\title{
Co-presentation of unilateral femoral and bilateral sciatic nerve variants in one cadaver: A case report with clinical implications
}

\author{
Patrick J Battaglia ${ }^{*}$, Frank Scali ${ }^{2}$ and Dennis E Enix ${ }^{1}$
}

\begin{abstract}
Objective: To present a group of anatomical findings that may have clinical significance.

Design: This study is an anatomical case report of combined lumbo-pelvic peripheral nerve and muscular variants.

Setting: University anatomy laboratory.

Participants: One cadaveric specimen.

Methods: During routine cadaveric dissection for a graduate teaching program, unilateral femoral and bilateral sciatic nerve variants were observed in relation to the iliacus and piriformis muscle, respectively. Further dissection of both the femoral nerve and accessory slip of iliacus muscle was performed to fully expose their anatomy.

Results: Piercing of the femoral nerve by an accessory iliacus muscle combined with wide variations in sciatic nerve and piriformis muscle presentations may have clinical significance.

Conclusions: Combined femoral and sciatic nerve variants should be considered when treatment for a lumbar disc herniation is refractory to care despite positive orthopedic testing.
\end{abstract}

Keywords: Sciatic nerve, Femoral nerve, Disc, Variant

\section{Background}

The recurrence of leg pain from lumbar disc herniations is a common post treatment clinical finding. Certain muscular and peripheral nerve variants may represent an unrecognized etiology in these cases and may affect the outcome of specific treatments. Recognition of these variations in normal anatomy may be useful to the clinician when treating the patient with refractory leg pain. The femoral nerve, derived from the second to fourth lumbar dorsal divisions, is one of the terminal branches of the lumbar plexus [1]. Multiple studies have reported variant slips of the psoas and iliacus muscles which may split the femoral nerve causing a potential risk for nerve entrapment [2-9]. In a large study of 121 cadavers, Vazquez et al. reported variations of iliacus and psoas muscles piercing the femoral nerve, piercing of the femoral nerve by a muscular slip, or a muscular slip/sheet

\footnotetext{
* Correspondence: patrick.battaglia@logan.edu

'Division of Research, Logan College of Chiropractic, 1851 Schoettler Rd, Chesterfield, MO 60317, USA

Full list of author information is available at the end of the article
}

covering the femoral nerve as it lay on the iliacus in 19 specimens (7.9\%) [3]. Several entities exist which may cause femoral neuropathy [10], however, owing to the lack of anatomic protection, entrapment of the nerve is most likely to occur immediately distal to the inguinal ligament [10]. No clinical finding is pathognomonic for femoral neuropathy, as similar findings of absent or diminished patellar reflex, quadriceps weakness or wasting, weakness in hip flexion and adduction as well as sensory symptoms such as pain in the iliac fossa, inguinal region, anterior thigh and medial calf may also indicate radiculopathy, plexopathy, or combined lesions of the femoral and obturator nerves [11].

The sciatic nerve, formed from the ventral rami of the fourth lumbar to third sacral spinal nerves, leaves the pelvis passing both anterior and inferior to the piriformis or sometimes through the muscle [1]. A 2010 literature review reported that the prevalence of piriformis and sciatic nerve variants in a large sample size of 6,062 cadaveric specimens was $16.9 \%$ [12]. The relationship of the piriformis and sciatic nerve causing piriformis

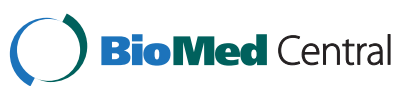


syndrome remains a controversial condition. Most commonly, sciatic neuropathy is iatrogenic, occurring after total hip arthroplasty [13]. Yuen, commenting on numerous studies, estimated the frequency of sciatica neuropathy after total hip arthoplasty to be between $0.7 \%$ and $3.7 \%$ [13]. Numerous other etiologies for sciatic neuropathy and sciatica pain exist $[13,14]$. Clinical exam findings of observed foot drop, and motor, sensory and reflex deficits in the sciatic nerve distribution are nonspecific for sciatic neuropathy and mimic lesions to the lumbosacral nerve roots or plexus [13].

Piercing of the femoral nerve by an accessory iliacus muscle in combination with bilateral variations in both sciatic nerve and piriformis muscle anatomy exemplifies the wide variability that exists within the lumbar and lumbosacral plexus. The clinical implications of these combined variants are discussed.

\section{Case presentation}

During routine cadaveric dissection, bilateral sciatic and unilateral femoral nerve variants were detected. The course and muscular relationships of both sciatic nerve variants were studied. The femoral nerve variant was further dissected and was examined to determine its nerve root contributions and its branching pattern. Also, the accessory muscular slip of the iliacus muscle that was piercing the femoral nerve was dissected to determine both its origin and insertion points.

On the right side, the sciatic nerve was split into the common fibular and tibial divisions proximal to the piriformis muscle, with the common fibular division passing above and superficial to the piriformis muscle and the tibial division passing inferior and deep to the muscle. On the left side, the sciatic nerve was also divided proximal to the piriformis muscle. However, the piriformis muscle was pierced and subdivided into two discrete bellies by the common fibular division, while the tibial division passed inferior and deep to the most caudal border of the piriformis muscle [Figure 1]. The right piriformis was one discrete muscle. On both sides the remaining course and distribution of the tibial and common fibular nerves was considered normal.

In the left iliac fossa, the femoral nerve emerged both lateral and deep to the psoas major muscle between the psoas major and iliacus muscles covered in iliac fascia. It was then pierced and divided into two separate divisions by an accessory slip of the iliacus muscle. Just proximal to the inguinal ligament, these two separate divisions rejoined and the femoral nerve passed as one under the inguinal ligament and then divided into its usual anterior and posterior branches [Figure 2]. The accessory slip of iliacus muscle was then dissected proximally up to its origin on the inferior aspect of the iliac crest. It was detached from its origin confirming it had no attachment to the iliolumbar ligament. The muscular slip was then followed distally until it blended into other iliacus and psoas major fibers to incorporate into the iliopsoas muscle which inserted on the lesser trochanter of the femur. The psoas minor and major muscles were then reflected to expose the lumbar plexus. The femoral nerve was found to be formed from the posterior division of the L2, L3 and L4 ventral nerve roots and was

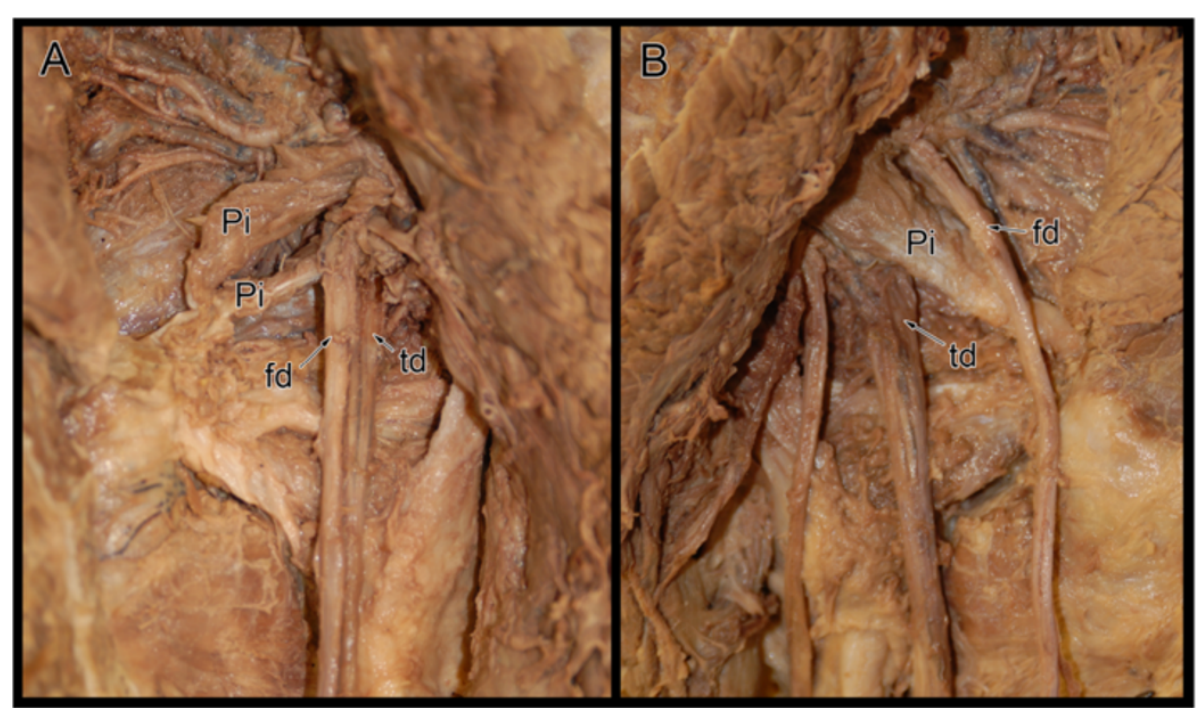

Figure 1 Photograph reveals bilateral sciatic nerve variants on a single cadaveric specimen. The left side (A) revealed that sciatic nerve divided proximal to the piriformis muscle. The left common fibular division (fd) divided the piriformis (Pi) into two distinct bellies while the left tibial division (td) passed inferior and deep to the most caudal border of the piriformis muscle. On the right side (B), the sciatic nerve was split into the right common fibular (fd) and tibial (td) divisions proximal to the piriformis muscle (Pi). The right common fibular division passed superficial while the tibial division passed deep to the piriformis muscle. 


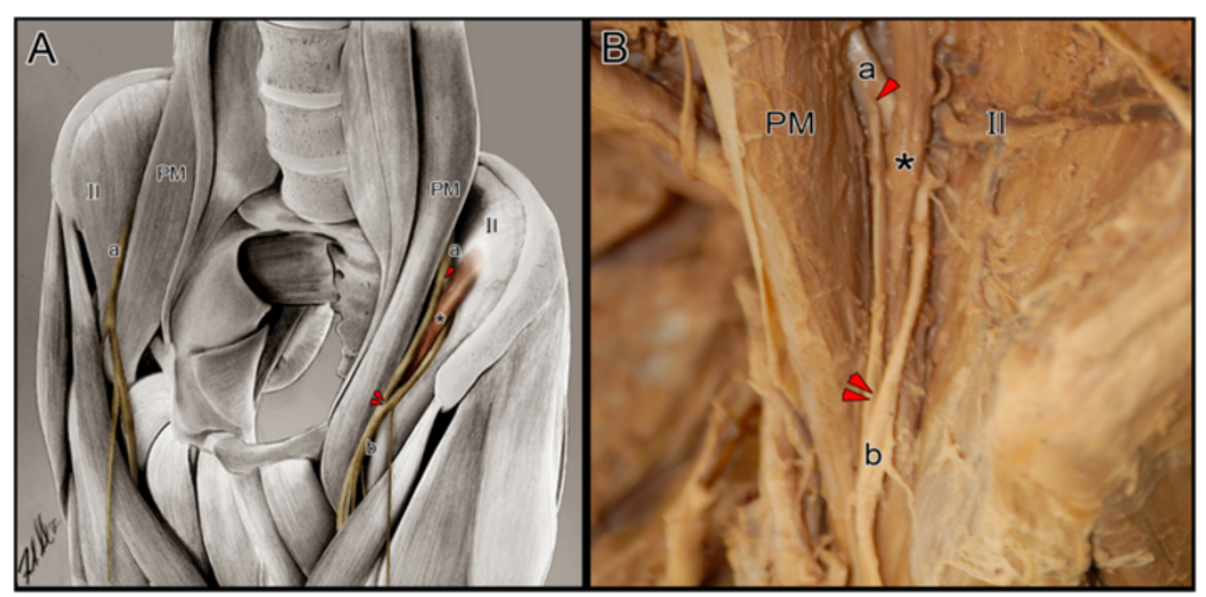

Figure 2 Illustration (A) and photograph (B) reveals unilateral (left side) femoral nerve variant. The femoral nerve (a) emerged bilaterally lateral and deep to the psoas major muscle (PM). While the right femoral nerve maintained its usual path, the left femoral nerve was pierced (arrowhead) and divided into two separate divisions by an accessory slip of the iliacus muscle ${ }^{*}$ ). These two separate divisions of the femoral nerve converged (double arrowheads) into a single division (b) and divided into its usual branches. Also labeled bilaterally is the iliacus muscle (II). (Original anatomical illustration by Frank Scali, DC).

fully formed prior to being pierced by the accessory slip of the iliacus muscle. No other lumbar plexus variations were detected. The femoral nerve on the right side of the specimen followed a routine course.

\section{Conclusions}

The sciatic and femoral nerves represent the two largest peripheral collections of lumbar and sacral nerve roots [1]. There have been other cadaveric reports of variance in sciatic and femoral nerve as well as piriformis and iliopsoas complex muscle anatomy similar to what is described in this case $[2-9,12,15]$. However, to the authors' knowledge, these variants have yet to be reported in one single specimen, and thus the potential clinical significance of these sole variants may be enhanced when possessed together.

Straight leg raise and femoral nerve traction tests are commonly performed orthopedic maneuvers done to ascertain the presence of a lumbar disc herniation $[16,17]$. Femoral nerve traction testing has a reported sensitivity of $50 \%$ and specificity of $100 \%$ for the diagnosis of midlumbar nerve root impingement, and appears to be insensitive and only $50 \%$ specific for lower lumbar nerve root impingement [18]. Straight leg raise testing has sensitivity and specificity characteristics of $16 \%$ and $31 \%$ respectively for midlumbar nerve root impingement. For the diagnosis of lower lumbar nerve root impingement, straight leg raise testing is $69 \%$ sensitive and $84 \%$ specific [18]. Reproduction of radicular leg pain in both sciatic and femoral nerve distributions with nerve traction testing is a common sign of lumbar disc herniations [16-18], and variations in both the course of the sciatic and femoral nerves as well as the surrounding musculature may affect the results of these nerve traction tests $[2,4]$.
Recovery from radicular symptoms is often problematic and may be due to diagnostic problems in challenging cases. In a retrospective study conducted by Suri et al. in 2012, $81 \%$ of patients who sought conservative care for their leg pain associated with a lumbar disc herniation experienced resolution of symptoms in an average of 6 months. However, within 1 year post resolution, 25\% had experienced a recurrence in their leg pain [19]. Patients who are refractory to care may warrant a reexamination, keeping in mind the many variations in anatomy, dermatomal patterns, and false positive/negatives of certain orthopedic tests [18,20-22]. A study of the distribution of dermatomal pain patterns by Murphy et al. showed $64.1 \%$ of the 169 lumbar spine pain patients presented with non-dermatomal pain distributions [23]. The sensitivity and specificity of lumbar spine dermatomal pain patterns associated with radiculopathies is too low to be useful in the identification of a specific nerve root level [23]. This is likely due to the communications between posterior collateral sensory ganglia and preganglionic neurons of different nerve root levels, creating variations in cutaneous sensations [22,24]. Several authors have concluded that variant femoral or sciatic nerve anatomy may produce a clinical picture analogous to that of a lumbar or lumbosacral radiculopathy $[2,4,5,7,14]$. Consideration of these anatomical variants, especially combined femoral and sciatic nerve variants, may prompt earlier or more focused diagnostic tests when a suspected lumbar spine disc herniation is refractory to care. One such test that may prove helpful to clinicians would be needle electromyography, as it can assist in the differentiation of radiculopathy and entrapment neuropathies [11]. 
Variants in lumbar and lumbosacral plexus anatomy should be considered when a symptomatic lumbar disc herniation is refractory to care. Recognition of these anatomical variants may lead to earlier intervention of physiologic testing, better treatment outcomes and improved patient satisfaction. Future studies examining the prevalence of these combined variants in the general population would be of interest to clinicians.

\section{Consent}

Written informed consent was obtained from the deceased prior to the gift of body donation. All handling of anatomical specimens was in accordance with the institutions ethical policy for body donation for anatomical study and scientific purposes. A copy of the written consent is available for review by the Editor-in-Chief of this journal.

\section{Competing interests}

The authors declare that they have no competing interests.

\section{Authors' contributions}

PB conceived of the case report, assisted in reviewing the literature and drafting the manuscript. FS provided anatomical artwork, assisted in reviewing the literature and drafting the manuscript. DE assisted in reviewing the literature, drafting the manuscript and provided critical review. All authors read and approved the final manuscript.

\section{Acknowledgements}

The authors would like to thank Robbyn Keating for her assistance in reviewing the literature.

\section{Author details}

'Division of Research, Logan College of Chiropractic, 1851 Schoettler Rd, Chesterfield, MO 60317, USA. ${ }^{2}$ Independent Researcher, 1272 Dutch Broadway, Valley Stream, NY 11580, USA.

Received: 14 July 2012 Accepted: 17 October 2012

Published: 29 October 2012

\section{References}

1. Standring S: Gray's Anatomy. The Anatomical Basis of Clinical Practice. 40th edition. New York: Elsevier Churchill Livingstone; 2008.

2. Spratt JD, Logan BM, Abrahams PH: Variant Slips of Psoas and Iliacus Muscles, With Splitting of the Femoral Nerve. Clin Anat 1996, 9:401-404.

3. Vazquez MT, Murillo J, Maranillo E, Parkin IG, Sanudo J: Femoral Nerve Entrapment: A New Insight. Clin Anat 2007, 20:175-179.

4. Fabrizio PA: Anatomic variation of the iliacus and psoas major muscles. Int J Anat Var 2011, 4:28-30.

5. D'Costa S, Ramanathan LA, Madhyastha A, Nayak SR, Prabhu LV, Rai R, Saralaya W, Prakash: An accessory iliacus muscle: a case report. Rom J Morphol Embryol 2008, 49(3):407-409.

6. Anloague PA, Huijbregts P: Anatomical variation of the lumbar plexus: A descriptive anatomy study with proposed clinical implications. J man manip ther 2009, 17(4):107-114.

7. Rao TR, Vanishree, Kanyan PS, Rao S: Bilateral variation of iliacus muscle and splitting of femoral nerve. Neuroanatomy 2008, 7:72-75.

8. Jelev L, Shivarov V, Surchev L: Bilateral variations of the psoas major and the iliacus muscles and presence of an undescribed variant muscle - accessory iliopsoas muscle. Ann Anat 2005, 187:281-286.

9. Astik RB, Dave UH: Anatomical variations in formation and branching pattern of the femoral nerve in iliac fossa: A study of 64 human lumbar plexuses. People's Journal of Scientific Research 2011, 4(2):14-19.

10. Chhabra A, Faridian-Aragh N: High resolution 3-T MR neurography of femoral neuropathy. Am J Roentgenol 2012, 198:3-10.

11. Busis NA: Femoral and obturator neuropathies. Neurol Clin 1999, 17(3):633-653.
12. Smoll NR: Variations of the piriformis and sciatic nerve with clinical consequence: a review. Clin Anat 2010, 23(1):8-17.

13. Yuen EC, Yuen TS: Sciatic neuropathy. Neurol Clin 1999, 17(3):617-631.

14. Stafford MA, Peng P, Hill DA: Sciatica: a review of history, epidemiology, pathogensis, and the role of epidural steroid injection in management. Br J Anaesth 2007, 99:461-73.

15. Khan YS, Khan TK: A rare case of bilateral high division of sciatic nerve (of different types) with unilateral divided piriformis and unusual high origin of genicular branch of common fibular nerve. Int J Anat Var 2011, 4:63-66.

16. Cram RH: Sign of sciatic nerve root pressure. J Bone Joint Surg 1953, 35-B:192-5.

17. Dyck P: The femoral nerve traction test with lumbar disc protrusions. Surg Neurol 1976, 3:163-6.

18. Suri P, Rainville J, Katz JN, Jouve C, Hartigan C, Limke J, Pena E, Li L, Swaim B, Hunter DJ: The Accuracy of the Physical Examination for the Diagnosis of Midlumbar and Low Lumbar Nerve Root Impingement. Spine 2011, 36(1):63-73

19. Suri P, Rainville J, Hunter DJ, Li L, Katz JN: Recurrence of radicular pain or back pain after nonsurgical treatment of symptomatic lumbar disc herniation. Arch Phys Med Rehabil 2012, 93:690-5.

20. van der Windt DA, Simons E, Riphagen II, Ammendolia C, Verhagen AP, Laslett M, Devillé W, Deyo RA, Bouter LM, de Vet HC, Aertgeerts B: Physical examination for lumbar radiculopathy due to disc herniation in patients with low-back pain. Cochrane Database Syst Rev 2010, 17(2):CD007431.

21. Lee MWL, McPhee RW, Stringer MD: An Evidence-Based Approach to Human Dermatomes. Clin Anat 2008, 21:363-373.

22. Greenberg SA: The history of dermatome mapping. Arch Neurol 2003 60:126-131.

23. Murphy DR, Hurwitz EL, Gerrard JK, Clary R: Pain patterns and descriptions in patients with radicular pain: Does the pain necessarily follow a specific dermatome. Chiro \& Osteo 2009, 17:9.

24. Cramer G, Darby S: Basic and Clinical Anatomy of the Spine, Spinal Cord, and ANS. 2nd edition. St. Louis: Elsevier Mosby; 2005.

doi:10.1186/2045-709X-20-34

Cite this article as: Battaglia et al:: Co-presentation of unilateral femoral and bilateral sciatic nerve variants in one cadaver: A case report with clinical implications. Chiropractic \& Manual Therapies 2012 20:34.

\section{Submit your next manuscript to BioMed Central and take full advantage of:}

- Convenient online submission

- Thorough peer review

- No space constraints or color figure charges

- Immediate publication on acceptance

- Inclusion in PubMed, CAS, Scopus and Google Scholar

- Research which is freely available for redistribution 